\title{
Teas: Direct test on quality and antioxidant activity using electron paramagnetic resonance spectroscopy
}

\author{
M.A. Morsy \\ Department of Chemistry, King Fahd University of Petroleum \& Minerals, Dhahran 31261, \\ Saudi Arabia
}

\begin{abstract}
The manufactured teas may conveniently be divided into three classes: Green teas, Oolongs (delicate black teas), and Black Teas. Several indirect methods are employed to measure the antioxidant activity of tea extracts that is basically takes place by monitoring the inhibition of oxidation of a suitable substrate using the extracts of antioxidant materials from the studies systems. This study is concerned with the results of electron paramagnetic resonance (EPR) spectroscopy that is utilized to investigate several categories of green and black teas: Twining Green Tea (TGT) of London, Rabea Green Tea (RGT) of Saudia, Chinese Green Tea (CGT) of China, English Breakfast Tea (EBT) of Ahmed Tea-London, and Rabea Black Tea (RBT) of Saudia. Three EPR signals from all the studied samples are observed. The assignment of these signals will discussed and correlated with the quality of leaves. Mainly, two paramagnetic species are considered to be responsible for the observed EPR signals; manganese(II)-protein system of the leaves and a stable free radical of aromatic origin. The study will provide facts on the dependency of radical signal on the oxidative degradation procedures of the studied samples. The intensity and feature of $\mathrm{Mn}(\mathrm{II})-\mathrm{EPR}$-signal is found to be related to the disintegration of tea leaves.
\end{abstract}

Keywords: EPR, green tea, black tea, antioxidants, manganese(II), quinone-radicals

\section{Introduction}

China and Japan give large portions of the tea crop that is intended for export to foreign countries. Tea leaves in these countries are used as a daily beverage and crude medicine for thousands of years. Commonly, tea is the most widely consumed drink in the world. The nutritional and therapeutic roles of green tea have been reviewed by Hara et al. [1]. Many pharmacological effects of tea are also evaluated [1-5] including antitumor properties, antioxidant, antigenotoxic and anticarious activities as well as the effect on cardiovascular and cerebrovascular diseases; it is also correlated with significant decrease in blood cholesterol levels.

Recently, tea and green tea in particular is considered as an effective source of antioxidants that attracted much attention [6,7]. Green tea extracts showed enhanced antioxidant activity in vegetable oils and animal fats [8-10]. For example, epigalloepicatechin gallate was found $[2,11]$ to be 20 times more potent than ascorbic acid derivatives which have been recently utilized as alternatives to the most widely used antioxidants in the food industry [12]; butylated hydroxyanisole (BHA) [13,14].

Several methods are employed to measure the antioxidant activity of tea extracts. Fore example, the extracts antioxidant effectiveness is measured by monitoring the inhibition of oxidation of a suitable substrate. After the substrate is oxidized under standard conditions, the extent of oxidation is measured by chemical, instrumental or sensory methods [15-18].

In this study we extend our earlier EPR spectroscopy studies utilized to monitor the generation and status of free radicals and paramagnetic species in natural fresh or partially fermented leaves [19-22]. 
It will be used to examine the antioxidation activities of different categories of tea products namely: Twining Green Tea (TGT) of London, Rabea Green Tea (RGT) of Saudia, Chinese Green Tea (CGT) of China, English Breakfast Tea (EBT) of Ahmed Tea-London, and Rabea Black Tea (RBT) of Saudia. The resistance against photooxidation damage was correlated by the increase of a specific sharp EPR signal characteristic of semiquinones-type radical, and the lineshape changes of a broad EPR signal characteristic of $\mathrm{Mn}(\mathrm{II})$. Moreover, the intensity of manganese(II) EPR signal is found to be related to ageing and disintegration of the tea leaves.

\section{Experimental procedures}

All categories of the tealeaves used in this study purchased at the local market. About $1 \mathrm{~cm}$ height of the studied samples was placed in $3 \mathrm{~mm}$-Pyrex tube. This amount of samples has an approximate mass of about $0.13 \mathrm{~g}$.

The EPR measurements were performed on the different types of the tea leaves as received and without any further treatment. ER-200-D-SRC series spectrometer was used to obtain the EPR spectra. Cylindrical cavity has been used along with $9 \mathrm{GHz}$ Microwave Bridge. The frequency was measured with a model 5342A Hewlett-packard frequency counter. The working parameters of EPR Bruker spectrometer are as follows: time constant $=655.36 \mathrm{~ms}$, mod. frequency $=25.0 \mathrm{kHz}$, mod. amplitude $10.0 \mathrm{G}$, frequency $9.60 \mathrm{GHz}$, resolution 2048 points, signal gain $1 \times 10^{4}$, sweep width $4400 \mathrm{G}$, sweep time $167.75 \mathrm{~s}$, and sample temperature $22^{\circ} \mathrm{C}$

The photodegradation experiments were performed using the Illuminator Power Supply from Varian Model PS150-8. The EPR tubes containing the different types of tealeaves were illuminated by xenon lamp at constant current (8 Amp DC) for different intervals of time.

\section{Results and discussion}

Figure 1 shows the recorded EPR spectra at room temperature of different types of the studied tealeaves as received. All of the samples under investigation were recently collected from the local market and their production date is around 01/2002 except for the old twinning green tea that is produced around 01/2000.

All the studied samples exhibit broad signals that are positioned around $3400 \mathrm{G}$ and are extended over $\sim 500 \mathrm{G}$ (see Fig. 1). The $g$-factor corresponding to the broad line in Fig. 1(a) is 2.02489. A weak sharp signal is also observed in the middle of these lines with a width of about $10 \mathrm{G}$ and $g$-factor value of 2.00023. These EPR-parameters and the featureless property of the sharp signal indicate that a stable radical of aromatic origin may exist in a powder condition. Similar signal was observed in a tar matrix and is attributed to semiquinone radical [23,24]. It has also been reported [25-27] that the macromolecular dihydric phenolic matrices from the natural leaves stabilize semiquinone free radicals which are simply examined by EPR spectroscopy. The overlapped sextet feature exhibited by some of the studied system is a characteristic EPR-signal of $\mathrm{Mn}$ (II) that is partially unbounded to proteins [28-30]. Moreover, in the course of the thorough examination of the EPR signal of TGT and CGT samples, a distortion at lower magnetic fields is observed. This distortion at lower magnetic field usually takes place whenever there are asymmetries in the electronic environment of the bonded Mn(II) [29]. Computer simulations suggest that this spectral line arises from rhombic distortions of the bound Mn(II) ion [29].

EPR-spectrum of $\mathrm{Mn}(\mathrm{II})$ is readily recognized in biological materials by its six hyperfine lines centered around $g=2$ [31]. Manganese(II) is an essential element and micronutrient in photosynthetic 


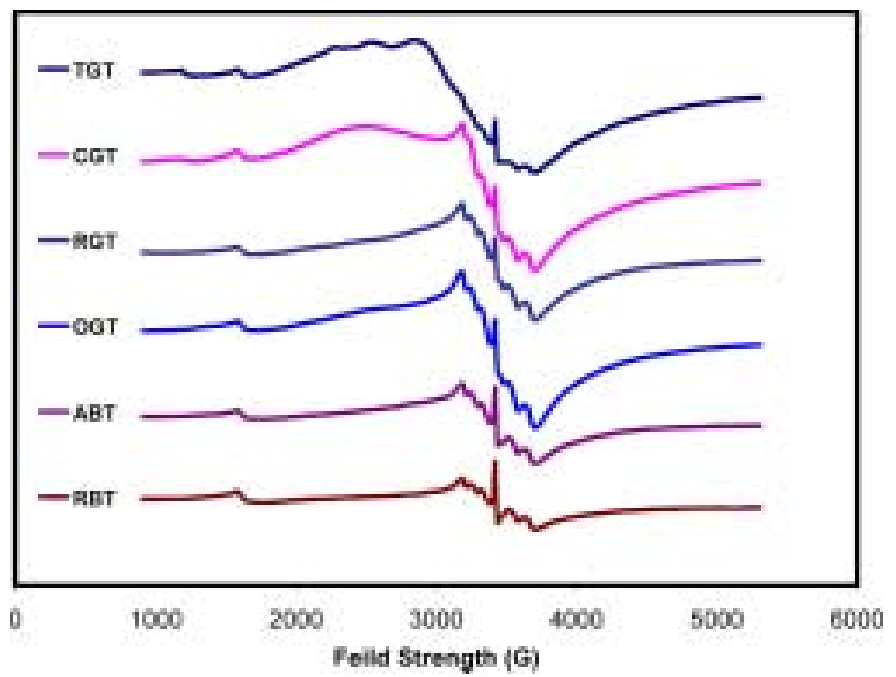

Fig. 1. EPR spectra of Twinning Green Tea (TGT), China Green Tea (CGT), Rabea Green Tea (RGT), Old Twinning Green Tea (OGT), Ahmed Breakfast Tea (ABT), and Rabea Black Tea (RBT) at room temperature.

green plants and cyanobacteria. It plays a fundamental role in the catalysis of water splitting and oxygen evolution in photosystem(II) which is a vital part of the plant survival [32,33].

The least featured broad signal of TGT in Fig. 1 is typical unresolved broad EPR line of a powder transition metal paramagnetic species [34]. The patterns in the EPR-signals of the tealeaves compared to the high quality tobacco leaf signal (Fig. 1(a) in [19] and [20]) indicate that the tealeaves undergo partial $\mathrm{Mn}$ (II)-protein degradation but with different extent. However, the main features of TGT EPR-signal relative to the others in Fig. 1 and to the reported $[19,20]$ high quality tobacco leaves placed TGT as the best quality tealeaf.

Close examination of the spectral lines in Fig. 1 shows an increase in the resolution of the Mn(II)sextet lines from parts RGT to RBT. The resolved broad hyperfine sextet in the tealeaves samples may be attributed to the free tumbling of manganese. It has been shown $[35,36]$ that the X-band EPR spectrum of the complex of Mn(II)-protein namely ATPase exhibit a powder line shape. These experiments showed also that $\mathrm{Mn}$ (II) bound to other macromolecules namely ATP, ADP, ANP-BNP increased the line broadening of the $\mathrm{Mn}(\mathrm{II})$-protein spectrum. In addition, the possibly related semiquinones signal is remarkably lower in magnitude in TGT compared to the other types of tealeaves.

The RBT in Fig. 1 is the one that exhibit most resolved sextet EPR signal among all the studied systems and shows the most intense semiquinones signal. The presence of a well resolved sextet and an intense quinone-free radical signals reflect the presence of partial unbounded $\mathrm{Mn}$ (II) as well as protein degradation, respectively. Protein degradation is due to the well known industrial treatment of the black tea, namely, longer fermentation process than green tea that induced structural changes in the Mn(II)protein. This result is in agreement with the reported [19] results of the Red-Label black tea (RLB-tea) where both of them approach the spectrum of free manganese tumbling. Similarity between the EPR responses from boiled tea-leaf and aqueous solutions of manganese(II) nitrate confirm this $\mathrm{Mn}$ (II)-freeing mechanism.

Figure 2 shows some of the EPR spectra of TGT after illumination by a xenon lamp for $0,15,30$, $45,60,75,90,105,120,150,210,270,390$, and 590 minutes. In this study a thorough investigation of the photodegradation processes is conducted to quantify the effect on the different samples. Over the ten 


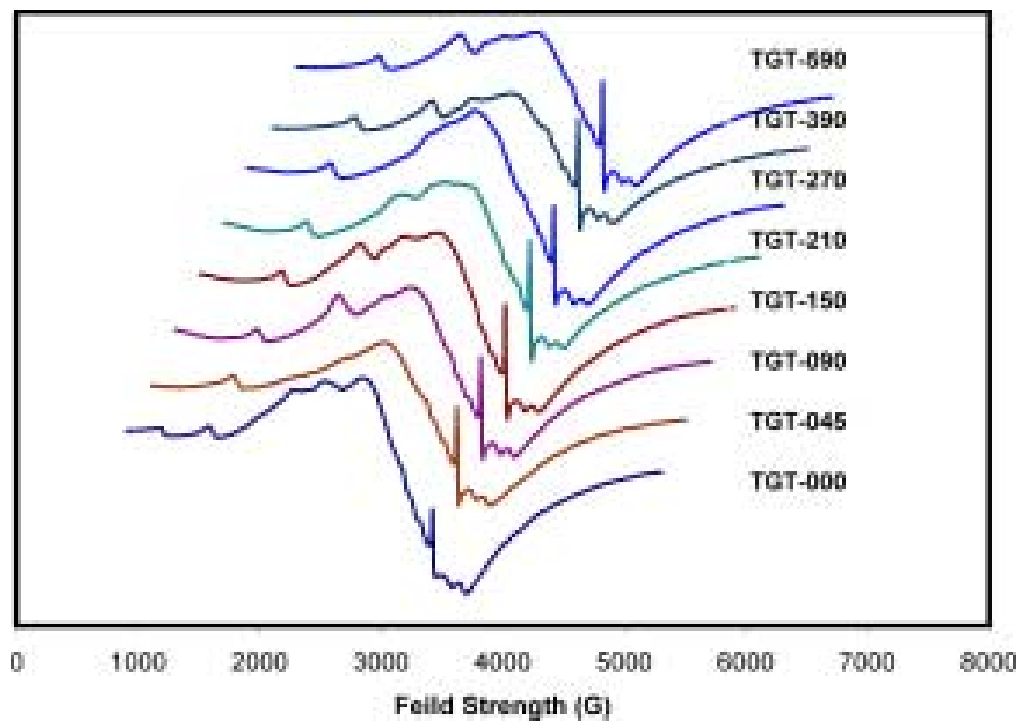

Fig. 2. EPR spectra of illuminated Twinning Green Tea (TGT) by xenon lamp at different time intervals in min.

hours of illumination the sextet remains almost unaffected but the distortion at the lower magnetic fields clearly affected that is related to rhombic distortions of the bound Mn(II) ion [29]. Moreover, a slight increase in the sharp signal with the duration of illumination after ten hours of continuous irradiation is noticed. Although the increase in the sharp signal of the quinone-radical is not significant in green tea even after 10 hours illumination, the distortion in the $\mathrm{Mn}$ (II) signal may be correlated with the startup of the damage in the protein center. This effect is strongly correlated with the result of the period of illumination and the intensities of the Mn(II)-signal per gram of the studied samples (Fig. 3). The results in Fig. 3 show a strong effect of the period of illumination on the green tea leaves types than the black tea leaves.

Although the $\mathrm{Mn}(\mathrm{II})$-signal of the RBT is not affected that much compared to the green tealeaves as shown in Fig. 3, its photo-oxidative result is more clearer. Figure 4 shows the EPR spectra of RBT under illumination for similar time intervals applied on green tea samples. As the photo-oxidation proceeds, remarkable increases in the magnitude of the quinones signal. Pryor et al. [25] and other researchers [37] suggested that the quinone-type radical consists of a $\mathrm{Q}-\mathrm{QH}_{2}$ system undergoing charge transfer interactions. The donor is a hydroquinone $\left(\mathrm{QH}_{2}\right)$ and the acceptor is a quinone $(\mathrm{Q})$. Moreover, quinones are very well known byproducts of oxidation of the phenolic systems in natural products and contribute to the browning and pigment formation $[38,39]$. Hence, the fermentation processes in black tea are correlated with a weak broad Mn(II)-signal (Fig. 3) and a relatively larger sharp signal of the quinone-radical as in Fig. 1 compared to the other types of tealeaves under investigation.

The general increase in the sharp signal can be correlated with the oxidation of the phenolic systems and the generation of the quinone-type radicals. To quantify the effect of photo-oxidation results for the studied systems a peak-to-peak height ratio of the quinone-signal relative to the manganese-signal entitled Relative Oxidation Intensity (ROI) is plotted versus time in Fig. 5 for both types of tealeaves. The ROI-value increase is more significant in the case of the black tea as well as ABT. The ROI-values of the black tea approaches a plateau after four hours illumination while the other types of tealeaves including RBT still show very week change. At the plateau the ROI-value of the black tea is about six time that of the green tea. The result is in very good agreement with other study comparing the antioxidant effects of 


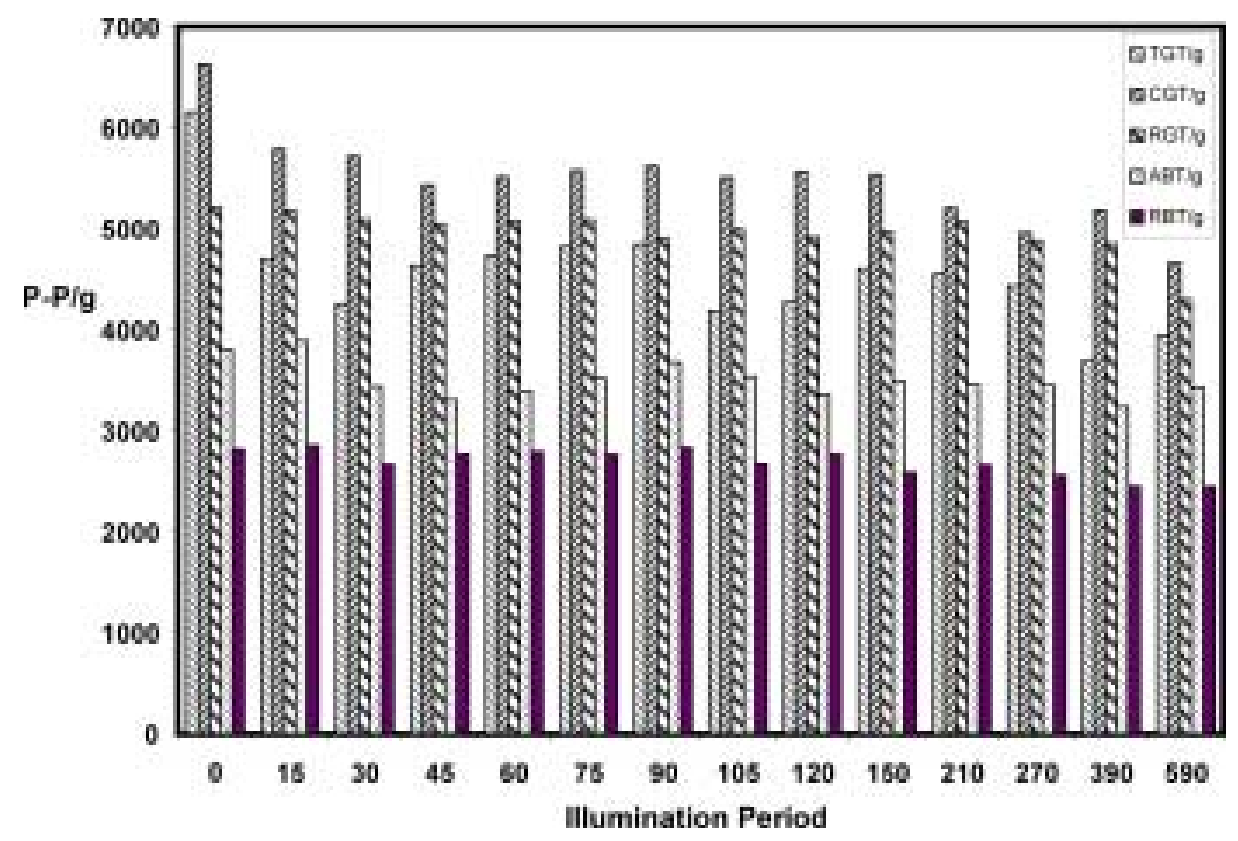

Fig. 3. Peak-to-peak height of the Mn(II)-signal per gram of tealeaves samples after the different period of illumination.

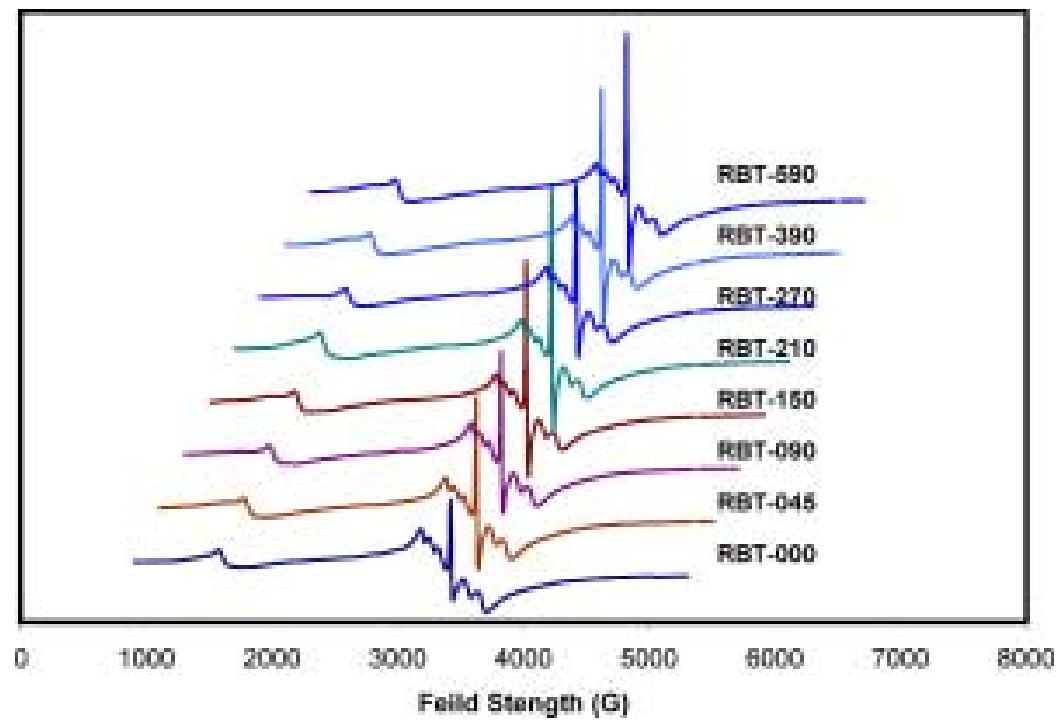

Fig. 4. EPR spectra of illuminated Rabea Black Tea (RBT) by xenon lamp at different time intervals in min.

green tea and black tea where the study indicated that the antioxidant effect of the green tea was found to be six times more potent than black tea [40]. This is also agree with the reported results on fermentation of green tea to black tea where the monomeric highly antioxidant polyphenols, known as flavonols, is oxidized to dimeric flavanols and polymeric polyphenols [12,41]. Results from Figs 3 and 5 show that $\mathrm{ABT}$ is midway type of tealeaf between the green and the black tea. 


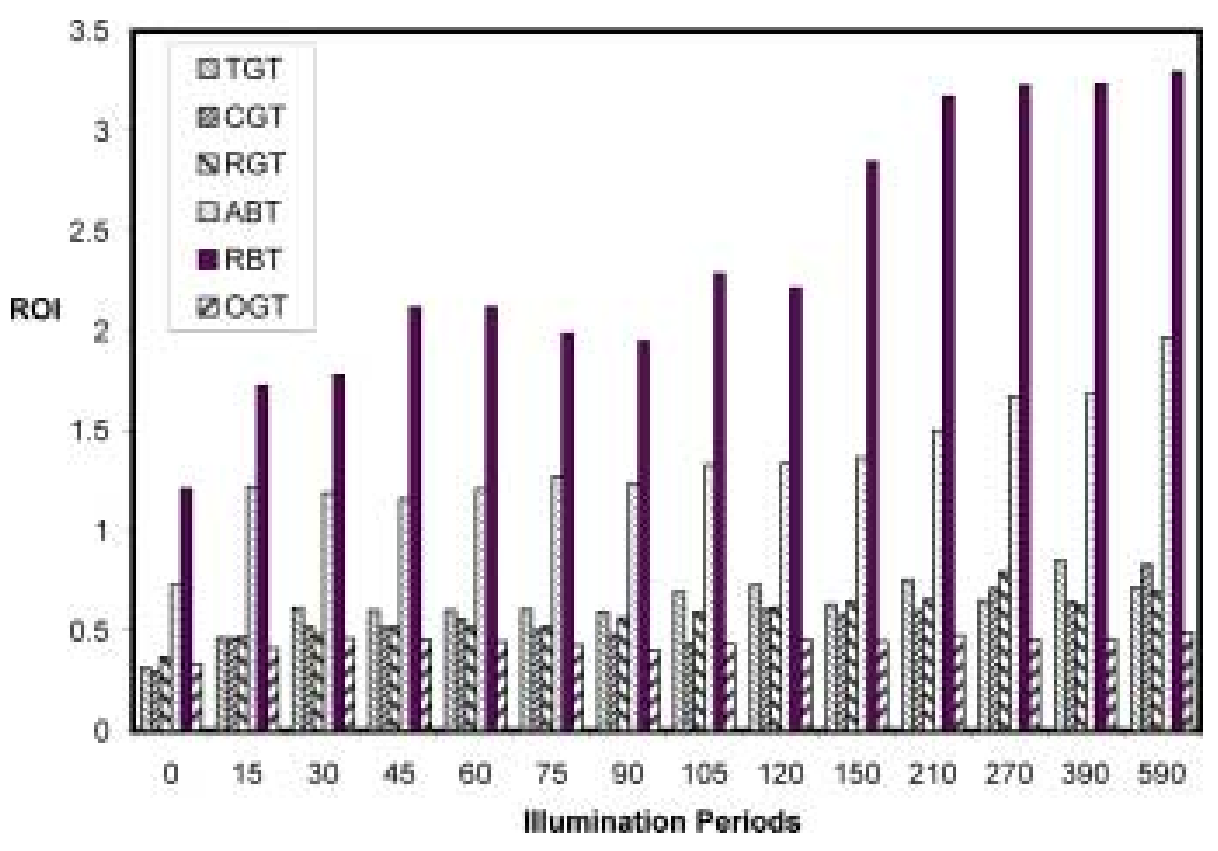

Fig. 5. Relative Oxidation Activity (ROA) for the illuminated types of tealeaves.

\section{Conclusion}

The three characteristic EPR signals from all the studied samples are characteristics of the presence of the manganese(II) complex in different environments and a stable radical of aromatic origin exist in a powder condition. The Mn(II) signal is found to be characteristic of the quality of leaf, industrial process and illumination. The increased features in the signal reflect more damage in the protein system and more of the free $\mathrm{Mn}$ (II) species. Distortion in the Mn(II) signal is also an indication on protein degradation. The more the degradation of the Mn-protein system the less the potent is the fermented leaves. Therefore, the manganese(II) EPR signal, according to this study and our earlier work [19,20], could be a mean of monitoring the freshness and quality of the fermented leaves.

The intensity of the aromatic-radical signal that may be related to semiquinone radical [23-27] is another parameter that can be used to quantify the damage in the protein system. The light irradiation experiment, therefore, shows that although some photo degradation takes place in the green tea namely, lowering in intensity as well as distortion at lower magnetic field which arises from rhombic distortions of the bound $\mathrm{Mn}(\mathrm{II})$ [29], it is still much more resistive to photo-oxidation. This correlates with the literature reports on the superior quality of the antioxidant phenols in green tea [6-9]. Moreover, the relative oxidation intensities of the black tea is six times stronger than the green tea which is in good agreement with other experiments performed chemical analysis on the same systems [40]. It is also observed in Fig. 5 that the quantified values of photo-oxidative effect of OGT shows a similar effect of the fresh green tea.

\section{Acknowledgement}

The authors acknowledge the support of King Fahd University of Petroleum and Minerals. 


\section{References}

[1] Y. Hara, S.-J. Luo, R.L. Wickremasinghe and T. Yamanishi, Chemical composition of tea, Food Rev. Int. 11 (1995), 435-456.

[2] T. Maatsuzaki and Y. Hara, Antioxidative activity of tea leaf catechins, J. Agric. Chem. Soc. Jap. 59 (1985), 129-134.

[3] S.K. Katiyar, R. Agarwal, G.S. Wood and H. Mukhtar, Inhibition of 12-O-tetradecanoyl phorbol-acetate caused tumerr promotion in 7,12-dimethybenz[a] anthracene initiated SEN-CAR mouse skin by a polyenolic fraction isolated from green tea, Cancer Res. 52 (1992), 6890-6897.

[4] G.C. Yen and H.Y. Chen, Comparison of antimutagenic effect of various tea extracts (green, oolong, pouching, and black tea), J. Food Prot. 57 (1994), 54-58.

[5] M.G.L. Hertog, P.C.H. Hollman and B. Putte, Content of potentially anticarcinogenic flavonoids of tea infusions, wins, and fruit juices, J. Agric. Food Chem. 41 (1993), 1242-1246.

[6] Y. Hara, Prophylactic functions of tea polyphenols, in: Food Phytochemicals for Cancer Prevention II. Teas, Spices, and Herbs, C.-T. Ho, T. Osawa, M.T. Huang and R.T. Rosen, eds, American Chemical Society, Washington, DC, 1994, pp. 3450 .

[7] N.F. Edwin, S.-W. Huang and R. Aeschbach, Antioxidant activity of green teas in different lipid systems, JAOCS 74 (1997), 10.

[8] Y. Hung, M.S. Vasyl, S.Yu. Makan, T.-H.J. Chen, M.-Y. Hong and G.S. Huang, Antioxidant activity of melanins derived from tea: comparison between different oxidative states, in: Food Chemistry 2002, in press, available online 3 January.

[9] P. Zandi and H.G. Michael, Antioxidant activity of extracts from old tea leaves, Food Chemistry 64 (1999), 285-288.

[10] D.A. Balentine, Manufacturing and chemistry of tea, in phenolic compounds, in: Food and Their Effects on Health I, C.-T. Ho, C.Y. Lee, M.T. Huang and C.Y. Huang, eds, American Chemical Society, Washington, DC, 1992, pp. 102-117.

[11] K. Michiko, N.G. Subhendu, J. Banerjee and A. Kobayashi, Aroma composition of oolong tea and black tea by brewed extraction method and characterizing compounds of Darjeeling tea aroma, J. Agric. Food Chem. 43 (1995), 200-207.

[12] Z.Y. Chen, P.T. Chan, H.M. Ma, K.P. Fung and J. Wang, Antioxidative effect of ethanol tea extracts on oxidation of Canola oil, JAOCS 73 (1996), 375-380.

[13] W.Z. Lin, S. Navaratnam, S.D. Yao and N.Y. Lin, Antioxidative properties of hydroxycinnamic acid derivatives and a phenylpropanoid glycoside - A pulse radiolysis study, Radiation Physics and Chemistry 53 (1998), 425-430.

[14] J.S. Jankun, H. Selman and R. Swierez, Why drinking green tea could prevent cancer?, Nature 387 (1997), 561.

[15] R. Kevin, P.D. Prenzler, G. Tucker, P. Swatsitang and W. Glover, Phenolic compounds and their role in oxidative processes in fruits, Food Chem. 66 (1999), 401-436.

[16] E.M. Marinova and N. Yamishlieva, Autooxidative activity of phenolic acids on triacylglycerols and fatty acid methyl esters from olive oil, Food Chem. 56 (1996), 139-145.

[17] M. Heinonen, D. Rein, M.T. Satue Gracia, S.W. Huang, J.B. German and E.N. Frankel, Effect of protein on the antioxidant ativity of phenolic compounds in a lecithin-liposome oxidation system, J. Agr. Food Chem. 46 (1998), 917-922.

[18] E.N. Frankel, In search of better methods to evaluate natural antioxidants and oxidative stability if food lipids, Trends in food Science and Technology 4 (1993), 220-225.

[19] M.A. Morsy and M.M. Khaled, Novel EPR characterization of the antioxidant activity of tea leaves, Spectrochim. Acta A 58 (2002), 1271-1277.

[20] M.A. Morsy and M.M. Khaled, Direct electron paramagnetic resosnance, study of tobacco. 1. Manganese(II) as a marker, J. Agric. Food Chem. 49 (2001) 683-686.

[21] M.A. Morsy and M. Khaled, EPR characterization of partially fermented natural leaves: Manganese (II) as a marker, in: 24th International Electron Paramagnetic Resonance Symposium, Denver, Colorado, USA, 2001.

[22] M. Khaled and M.A. Morsy, EPR detection and properties of naturally occurring free radicals in Mediterranean tobacco blends, in: 218th ACS National Meeting, New Orleans, LA, Division of Environmental Chemistry 39 (1999), 50-53.

[23] W.A. Pryor, B.J. Hales, P.I. Premovic and D.F. Church, The radicals in cigarette tar: Their nature and suggested physicological implications, Science 220 (1983), 425-427.

[24] E.T. Borish, J.P. Cosgrove, D.F. Church, W.A. Deutsch and W.A. Pryor, Cigarette tar causes single-strand breaks in DNA, Biochem. Biophys. Res. Comm. 133 (1985), 780-786.

[25] J.A. Pedersen, Distribution an taxonomic implications of some phenolics in the family Lamiaceae determined by ESR spectroscopy, Biochemical systematics and Ecology 28 (1999), 229-253, and references therein.

[26] A. Jezierski, F. Czechowski, M. Jerzykiewicz, Y. Chen and J. Drozd, Spectrochimica Acta A 56 (2000), 379 and references therein.

[27] A. Jezierski, F. Czechowski, M. Jerzykiewicz and J. Drozd, App. Mag. Res. 18 (2000), 127 and references therein.

[28] G. Reed and W. Ray, Electron paramagnetic resonance studies of manganese(II) coordination in the phosph-glucmutase system, Biochemistry 10 (1971), 3190-3197.

[29] G. Reed and M. Cohn, Electron paramagnetic resonance studies of manganese(II)-protein complexes, J. Biol. Chem. 245 (1970), 662-667. 
[30] J. Villafranca, D. Ash and F. Weldler, Manganese(II) and substrate interaction with unadenylated glutamine synthetase (Escherichia coli W). II. Electron paramagnetic resonance and nuclear magnetic resonance studies of enzyme-bound manganese(II) with substrate and potential transition-state analogue, methionine sukfoximine, Biochemistry 15 (1976), 544-553.

[31] H. Beinert and G. Palmer, Adv. Enzymol. 27 (1965), 105.

[32] R.J. Debus, The manganese and calcium ions of photosynthetic oxygen evolution, Biochim. Biophys. Acta 1102 (1992), $269-352$.

[33] G.W. Brudvig, W.F. Beck and J.C. De Paula, Mechanism of photosynthetic water oxidation, Ann. Rev. Biophys. Biophys. Chem. 18 (1989), 25-31.

[34] H.G. Hecht, Magnetic Resonance Spectroscopy, Wiley and Sons, Inc., New York, 1967, p. 131.

[35] C.M. Grisham, Manganese(II) and gadolinium(III) ESR studies of membrane bound ATPases, in: Polymer Characterization by ESR and NMR, ACS Symposium Series 142, A.E. Woodward and F.A. Borey, eds, Washington, DC, 1980, pp. 490-80, and references therein.

[36] C.M. Grisham, The structure of the $\left(\mathrm{Na}^{+}+\mathrm{K}^{+}\right)$-ATPase. Implications for the mechanism of sodium and potassium transport, in: Advances in Inorganic Biochemistry, G. Eichhron and L. Marzilli, eds, Elsevier/North-Holland, 1979, pp. 193235.

[37] I. Schmeltz, J. Tosk, G. Jacobs and D. Hoffmann, Redox potential and quinone content of cigarette smoke, Anal. Chem. 49 (1977), 1924-1929.

[38] F. Kader, J.P. Halluk, J.P. Nicolas and M. Metche, Degradation of cyanin 3-glycoside by blueberry polyphenol oxidasekinetic studies and mechanisms, J. Agr. Food Chem. 46 (1998), 060-065.

[39] M.J. Amiot, M. Tacchini, S.Y. Aubert and W. Oleszek, Influence of cultivar, maturity stage, and storage conditions on phenolic composition and enzymatic browning of pear fruits, J. Agr. Food Chem. 43 (1995), 1132-1137.

[40] M. Serafini, A. Ghiselli and A. Ferro-Luzzi, In vivo antioxidant effect of green and black tea in man, Eur. J. Clin. Nutr. 50 (1996), 28-32.

[41] Jifu Zhao, Xinho Jin, E. Yaping, Zai S. Zheng, Yu J. Zhang, Mohammad Athar, Vincent A. DeLeo, Hasan Mukhtar, David R. Bickers and Zhi Y. Wang, Protective effect of black tea extracts against UVB-induced toxicity in skin, Photochemistry and Photobiology 70 (1999), 637-644. 


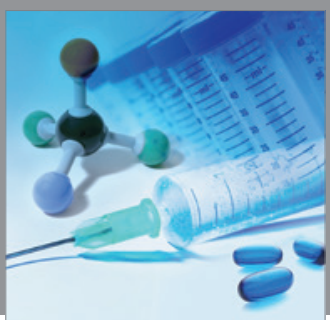

International Journal of

Medicinal Chemistry

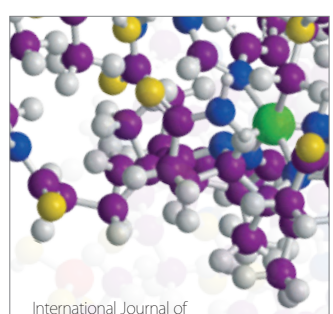

Carbohydrate Chemistry

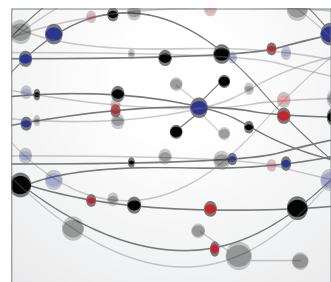

The Scientific World Journal
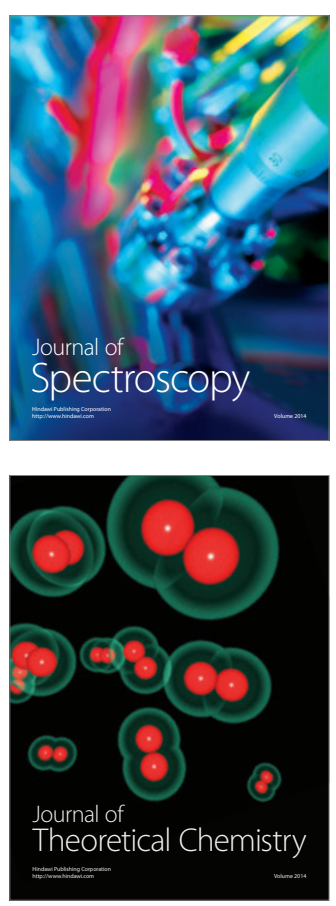
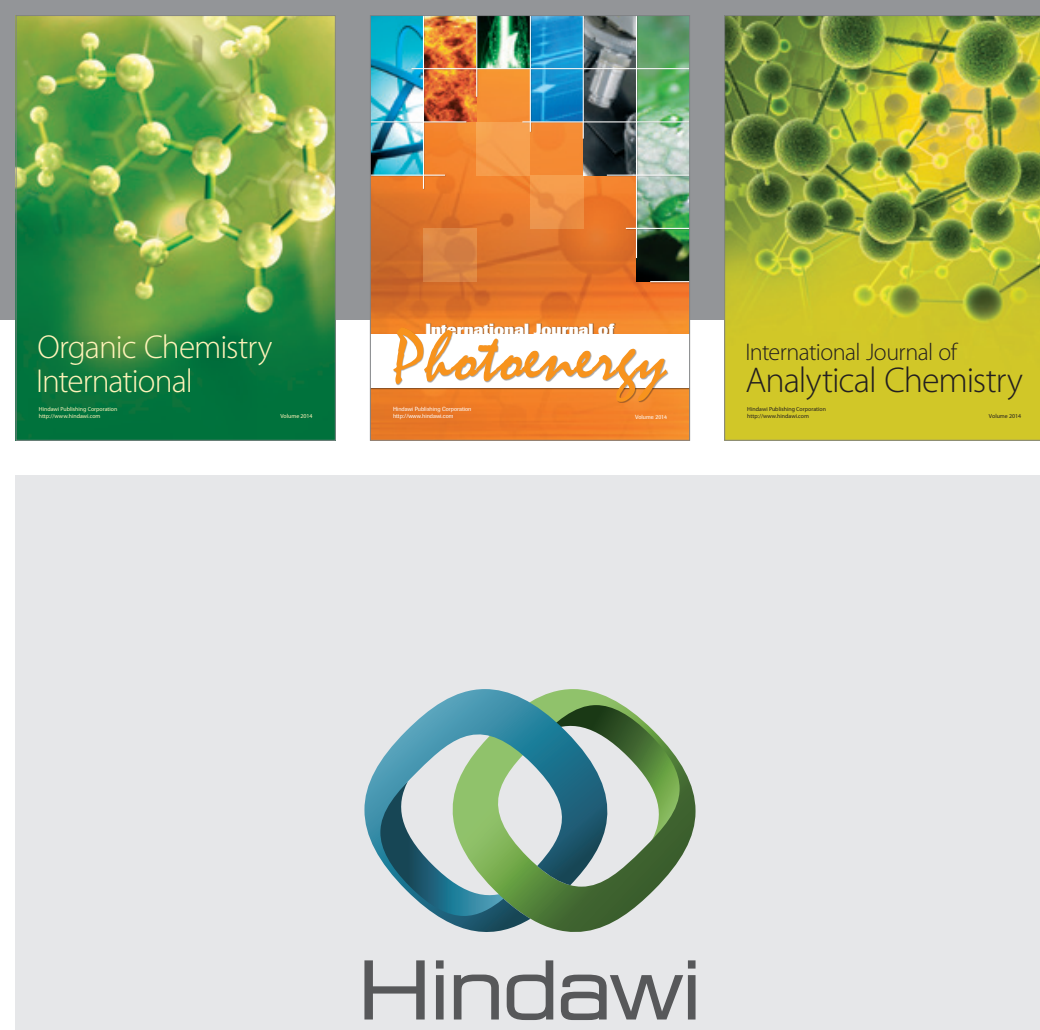

Submit your manuscripts at

http://www.hindawi.com
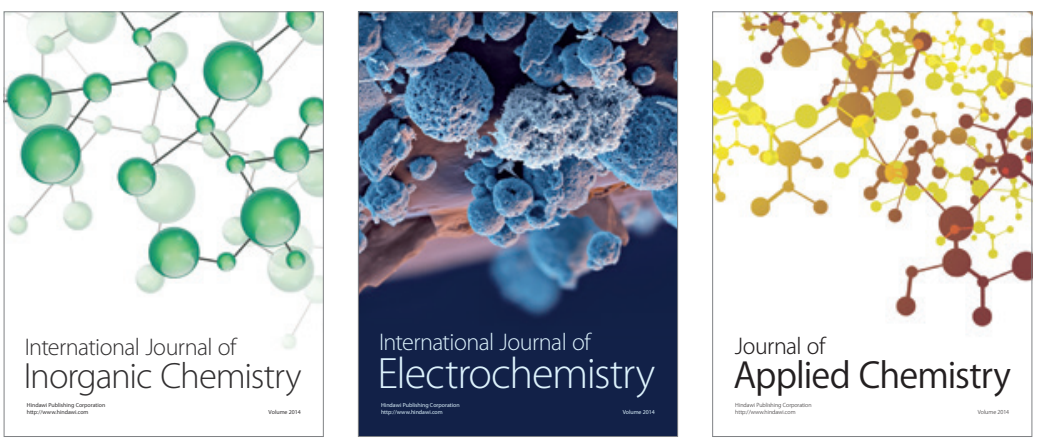

Journal of

Applied Chemistry
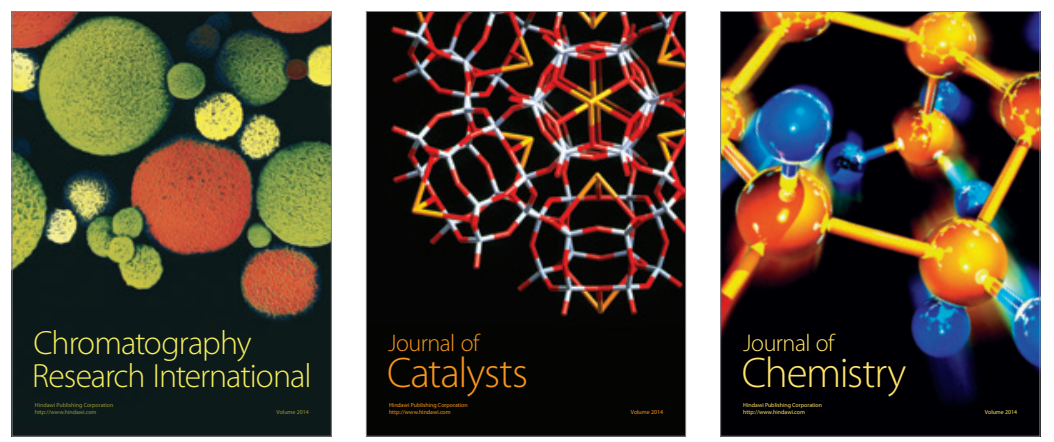
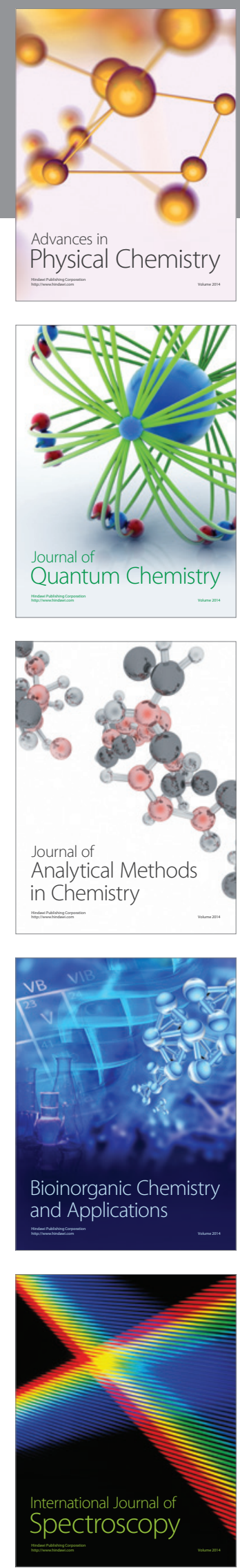